ISSN: 0212-5374

DOI: http://dx.doi.org/10.14201/et2014321195213

\title{
ACTITUDES DEL DOCENTE DE CENTROS DE EDUCACIÓN ESPECIAL HACIA LA INCLUSIÓN EDUCATIVA
}

\author{
Attitudes of teachers education centers to special education \\ inclusion
}

\section{Attitudes des enseignants des centres d'éducation au special inclusion de l'éducation}

M. ${ }^{a}$ del Carmen Pegalajar Palomino* y M. ${ }^{a}$ Jesús Colmenero Ruiz***

Universidad de Jaén (España). Correo-e: *mcpegala@ujaen.es; ***miruiz@ujaen.es

Recibido: 09.04.2013; Aceptado: 12.01.2013; Publicado: 31.12.2014

BIBLID [0212-5374 (2014) 32, 2; 195-213]

Ref. Bibl. M..$^{a}$ DEL CARMEN PEGALAJAR PALOMINO y M. a JESÚS COLMENERO RUIZ. Actitudes del docente de centros de Educación Especial hacia la inclusión educativa. Enseñanza \& Teaching, 32, 2-2014, 195-213.

RESUMEN: Esta investigación pretende analizar las actitudes y percepciones hacia la educación inclusiva del docente que desarrolla su actividad profesional en centros de Educación Especial de Andalucía. En el trabajo se ha empleado una metodología descriptiva, participando un total de 428 docentes y utilizando para la recogida de datos un cuestionario semiestructurado elaborado ad hoc. Los resultados obtenidos demuestran cómo los docentes de los centros de Educación Especial de Andalucía consideran la educación inclusiva como pilar básico en la educación, a la vez que definen la modalidad de escolarización en centros de Educación Especial como una respuesta educativa al alumnado tendente a la mejora de su calidad de vida y a la consecución de un mayor grado de autonomía personal y funcionalidad en las actividades de la vida diaria. Del mismo modo, consideran que el alumnado 
M. 므 DEL CARMEN PEGALAJAR PALOMINO Y M. ${ }^{a}$ JESÚS COLMENERO RUIZ ACTITUDES DEL DOCENTE DE CENTROS DE EDUCACIÓN ESPECIAL HACIA LA INCLUSIÓN EDUCATIVA

escolarizado en este tipo de centros educativos presenta mayores necesidades educativas en el ámbito de la salud, cognitivo y social. Se destaca, incluso, la existencia de diferencias estadísticamente significativas en las actitudes y percepciones de los docentes según éstos hayan desarrollado o no actividades de formación práctica vinculada al ámbito de la educación inclusiva durante su período de formación inicial, siendo más favorables en el caso de aquellos que no disponen de tal formación. De este modo, se pretende conocer las percepciones de estos docentes sobre la educación inclusiva para poder establecer propuestas de mejora en la formación del docente que lleven al desarrollo de un proceso de enseñanza y aprendizaje de calidad para el alumnado con discapacidades graves y permanentes.

Palabras clave: educación inclusiva, necesidades específicas de apoyo educativo, discapacidades graves y permanentes, centros de educación especial, actitudes, docente.

SUMMARY: This research aims to analyze the attitudes and perceptions towards inclusive education teacher that practices in special education centers in Andalusia. The paper has used a descriptive methodology, a total of 428 participating teachers and using data collection a semi structured questionnaire developed ad hoc. The results show how teachers of special education centers in Andalusia consider inclusive education as a pillar in education, while defining the mode of schooling in special education institutions as an educational response to students aimed at improving their quality of life and achieve greater personal independence and function in daily life activities. They also consider that the students enrolled in these schools has higher educational needs in the field of health, cognitive and social. It stands out even the existence of statistically significant differences in attitudes and perceptions of teachers as they have developed or no practical training related to the field of inclusive education during their initial training, being more favorable in the case of those without such training. Thus, we want to know the perceptions of these teachers on inclusive education to establish proposals for improvement in teacher education that lead to the development of a process of quality teaching and learning for pupils with severe and permanent disabilities.

Key words: inclusive education, specific educational needs, severe and permanent disabilities, special education centers, attitudes, teacher.

RÉSUMÉ: Cette recherche vise à analyser les attitudes et les perceptions à l'égard des enseignants d'éducation inclusive qu'elle pratiques dans les centres d'éducation spéciale en Andalousie. Le document a utilisé une méthodologie descriptive, un total de 428 enseignants participants et la collecte de données à l'aide d'un questionnaire semi-structuré développé ad hoc. Les résultats montrent comment les enseignants des centres d'éducation spéciale en Andalousie considérent l'éducation inclusive comme un pilier dans l'éducation, tout en définissant le mode de scolarisation dans les établissements d'éducation spéciale comme une réponse éducative aux élèves visant à améliorer leur qualité de vie et d'atteindre une plus grande autonomie 


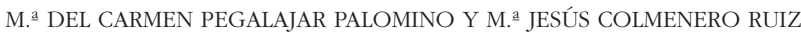

ACTITUDES DEL DOCENTE DE CENTROS DE EDUCACIÓN ESPECIAL HACIA LA INCLUSIÓN EDUCATIVA

personnelle et fonction dans la vie quotidienne. Ils considèrent également que les élèves inscrits dans ces écoles ont des besoins plus élevés d'éducation dans les domaines de la santé, cognitif et social. Il se distingue encore l'existence de différences statistiquement significatives dans les attitudes et les perceptions des enseignants qu'ont développé ou pas de formation pratique liée au domaine de l'éducation inclusive au cours de leur formation initiale, être plus favorable dans le cas de ceux qui n'ont pas une telle formation. Ainsi, nous voulons connaître les perceptions de ces enseignants sur l'éducation inclusive pour établir des propositions d'amélioration de la formation des enseignants qui conduisent à l'élaboration d'un processus de qualité de l'enseignement et de l'apprentissage pour les élèves ayant une déficience grave et permanente.

Mots clés: inclusive, besoins éducatifs spécifiques, déficience grave et permanente, centres d'éducation spéciale, les attitudes des enseignants.

\section{INTRODUCCIÓN}

En el actual sistema educativo, se plantean modelos educativos capaces de atender a la diversidad del alumnado desde un enfoque inclusivo. Los principios en que debe basarse dicho concepto suponen una participación más eficaz de los alumnos en el currículo, las culturas y las comunidades educativas; la reestructuración de las culturas, políticas y prácticas educativas (tanto en centros ordinarios como en centros de Educación Especial) para así dar respuesta a la diversidad del alumnado en su contexto; la presencia, participación y los resultados escolares de todos los alumnos vulnerables a las presiones exclusionistas, no sólo de aquellos con discapacidad o que estén catalogados como alumnos con necesidades educativas (Ainscow, Booth y Dyson, 2006). Este nuevo término pertenece al universo de la ética, la justicia social, la democracia y la equidad (Thomazet, 2009).

El nuevo modelo de atención a la diversidad se concreta en la práctica mediante una respuesta educativa adecuada a las características, posibilidades y necesidades del alumnado (Agran, Alper y Wehmeyer, 2002; Rosenberg, Westling y McLeskey, 2008). Así pues, y en el caso de alumnado con discapacidades graves y permanentes, los centros de Educación Especial suponen una modalidad de escolarización dirigida a conseguir los fines que la educación persigue para la generalidad del alumnado. Éstos deben apostar por optimizar el desarrollo del alumno hacia la mejora de la calidad de vida y la consecución de un mayor grado de autonomía y funcionalidad en las actividades de la vida diaria.

Tal y como se promueve en el Manual de Buena Práctica de Educación (FEAPS, 2000), los centros de Educación Especial deben tener un carácter altamente integrador, planteando compartir actividades y recursos con centros ordinarios, programas de intervención en la comunidad, apertura permanente al exterior y con una cultura de flexibilidad y creatividad continua. Ello supone la organización y ordenación de los recursos personales y materiales a la vez que el diseño 
M.․․ DEL CARMEN PEGALAJAR PALOMINO Y M..$^{\underline{a}}$ JESÚS COLMENERO RUIZ ACTITUDES DEL DOCENTE DE CENTROS DE EDUCACIÓN ESPECIAL HACIA LA INCLUSIÓN EDUCATIVA

e implantación de una oferta de actividades que favorezcan la inclusión y que sean normalizadoras según la edad del alumnado.

A pesar de los críticos que cuestionan la eficacia de la práctica inclusiva en el aula (Kavale, 2000; Kavale y Forness, 2000), cada vez con mayor frecuencia los profesores llevan a cabo en sus prácticas diarias estrategias de enseñanza inclusivas (Orr y Hamming, 2009). Así, existen algunos estudios que demuestran los efectos positivos de la educación inclusiva en alumnos con discapacidades graves y permanentes: Rafferty, Piscitelli y Boettcher (2003) o Bunch (2008) señalan la incidencia positiva del ambiente inclusivo en el proceso de aprendizaje del alumnado con discapacidad severa y, de igual modo, otros autores como Cole, Waldron y Majd (2004), Myklebust (2007) o Ruijs y Peetsma (2009) revelan cómo la motivación y los resultados académicos del alumnado con discapacidad aumentan en este tipo de contextos.

El hecho de admitir las diferencias entre el alumnado es algo relativamente fácil. La dificultad radica en tratarlas educativamente enriqueciendo el proceso de enseñanza-aprendizaje. La educación inclusiva requiere, por tanto, de una reestructuración y el desarrollo de importantes cambios en las organizaciones educativas y en el sistema en su conjunto para así responder a todo el alumnado (Raffo et al., 2009), dejando en un segundo plano el uso de métodos tradicionales de enseñanza (Salend, 1998; Lewis y Doorlag, 1999; Friend y Bursuck, 2002).

Tal y como han puesto de manifiesto Echeita y Simon (2007), para progresar en los modos de responder a las necesidades educativas de los alumnos con discapacidad en el contexto de una escuela inclusiva, no basta con apelar a la ética y a los valores en que dicha concepción descansa (Vlachaou, 2004; Booth, 2006) o a los potenciales efectos beneficiosos que reportaría al sistema educativo (Echeita, 2007), ni con presentar al profesorado nuevas concepciones, recursos o pautas de acción eficaces (Booth y Ainscow, 2000), sino que también y necesariamente hay que modificar las creencias implícitas al respecto mediante un proceso de explicitación progresiva de esas representaciones y de reorganización de las mismas en teorías más potentes y coherentes con los valores declarados. Como indica Forlin (2010), la educación inclusiva incide directamente en el sistema de valores del profesorado, cuestionando sus creencias más íntimas sobre lo que es correcto y justo.

Así pues, el análisis de las actitudes del docente hacia la educación inclusiva supone un tema de enorme interés en el ámbito de la Educación Especial. Así, por ejemplo, Hegarty (2008) realizó un recorrido sobre los temas o líneas de investigación en Educación Especial identificando al docente y sus actitudes como uno de los ámbitos prioritarios. Por su parte, Orcasitas (1994) contempló los estudios sobre actitudes hacia los procesos de integración en las instituciones educativas y no educativas como una de las líneas prioritarias de investigación. Otros autores como Cardona y Gómez (2001) apuntaron cómo la investigación en Educación Especial se encuentra en un estado inmaduro, por lo que se requiere de investigaciones para resolver problemas que aún persisten o que se han presentado, dirigidos a profundizar en las creencias y percepciones del profesorado acerca de la diversidad y de la respuesta educativa a la misma, entre otros. 
Sin embargo, y tal como establecen Boer, Jan y Minnaert (2011), algunos de los factores que influyen en la actitud de los maestros hacia la educación inclusiva y la atención a la diversidad son: la especialidad y la función docente, la formación inicial recibida, el conocimiento que se tiene, la edad y los años de experiencia. Como Hagger y Macintyre (2006) destacan "cualquier estudiante necesita para su desarrollo profesional aprender a ser docente en las escuelas, y es en esas escuelas donde necesitan aprender a hacer estas cosas" (p. 65). El Informe mundial sobre Discapacidad (OMs, 2011) puntualiza cómo «la adecuada formación de los docentes es crucial a la hora de ser competentes enseñando a niños con diversas capacidades" (p. 222) y enfatiza la necesidad de que dicha formación se centre en las actitudes y los valores, y no sólo en conocimientos y habilidades. Existen ciertas dimensiones en relación a los procesos eficaces de enseñanza que no pueden ser predichas por pruebas académicas (Menter et al., 2010). Investigaciones como las desarrolladas por Echeita et al. (2008) demostraron cómo los ámbitos de actuación en política educativa deberían concertarse en ampliar las posibilidades de formación de los profesores para atender con calidad a la diversidad del alumnado e incentivar procesos de mejora e innovación educativa en los centros educativos ordinarios, entre otros. Asimismo, cuanto menos competente se siente un profesor más actitudes negativas tiene, y eso repercute en una menor atención e interacción con los alumnos (Salazar, 2010).

Los resultados revelados por Ghanizadeh, Bahredar y Moeini (2006) muestran la existencia de una relación positiva entre el conocimiento de los maestros y su actitud hacia la educación inclusiva. Según un estudio de Batsiou et al. (2008) existe una relación significativa entre la información que los maestros tienen sobre la atención a la diversidad y las actitudes que muestran hacia la inclusividad; las actitudes docentes estaban influenciadas por la información y el conocimiento que los maestros tienen sobre la inclusión. Por su parte, Hsien (2007) ha mostrado cómo las actitudes del profesorado (tanto de la educación ordinaria como de la Educación Especial) respecto a la inclusión son, en general, positivas y dependen, ante todo, de la formación recibida en el manejo de las diferencias así como del sentimiento de competencia profesional. También Hammond y Lawrence (2003), Kim, Park y Snell (2005) o Batsiouet al. (2008) encontraron actitudes positivas en los docentes hacia la inclusión, entendiendo que ello beneficia a todos los alumnos.

No obstante, tanto Alghazo y Naggar (2004) como Avramidis y Kalyva (2007) indicaron que los maestros mostraban una actitud neutral o de indecisión hacia la inclusión de alumnos con necesidades educativas. Kalyva, Gojkovic y Tsakiris (2007), en un estudio de las actitudes de maestros de Primaria en Serbia, encontraron actitudes negativas hacia algunos aspectos fundamentales de la inclusividad en la enseñanza. Por ello, tal y como indica Gento (2006), con frecuencia los maestros no muestran una predisposición favorable hacia el tratamiento inclusivo de la diversidad, ya que "desconocen la forma de proceder adecuadamente y no están suficientemente informados de los recursos necesarios con los que pueden contar". 


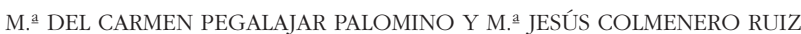
ACTITUDES DEL DOCENTE DE CENTROS DE EDUCACIÓN ESPECIAL HACIA LA INCLUSIÓN EDUCATIVA

\section{DisEÑO DE LA INVESTIGACIÓN}

\subsection{Objetivos}

El objetivo fundamental de nuestro estudio consiste en conocer y analizar las actitudes y percepciones del docente de los centros de Educación Especial de la comunidad autónoma andaluza hacia la educación inclusiva. En este sentido, se entiende que los centros de Educación Especial, lejos de considerarse una medida de segregación, deben apostar por plantear actividades y recursos compartidos con centros ordinarios, así como mantener una apertura permanente al exterior, cultura de flexibilidad y creatividad continua, favoreciendo el proceso de inclusión del alumnado con discapacidad grave y permanente en su contexto escolar y social. De igual modo, se pretende demostrar la existencia de diferencias estadísticamente significativas en las actitudes de los docentes según la formación práctica recibida sobre educación inclusiva durante su período de formación inicial.

Diversos autores (Fullan, 2002; Aguerrondo, 2004; Vaillant, 2005; Vezub, 2007) señalan al docente como elemento clave de transformación educativa y actor principal de la renovación de los modelos de enseñanza. Incluso, Imbernón (2004) destaca cómo "aunque parezca que lo sabemos casi todo acerca del profesorado, su desarrollo profesional y su formación, lo cierto es que su estilo se remonta a hace pocos años" (p. 125). Por ello, se intenta determinar cuáles son las áreas problemáticas que dificultan una respuesta educativa eficaz para el alumnado con necesidades educativas; su detección y valoración nos puede llevar a desarrollar importantes avances en la atención educativa prestada a personas con discapacidad.

\subsection{Metodología}

En esta investigación se ha utilizado un método descriptivo el cual pretende "describir sistemáticamente hechos y características de una población dado un área de interés de forma objetiva y comprobable» (Colás y Buendía, 1992: 177). Esta metodología es la adecuada cuando se desea hacer una exploración de un determinado fenómeno educativo para, a continuación, conocer esta realidad y poder mejorarla. Para ello, se ha elaborado un cuestionario ad hoc denominado "Cuestionario sobre necesidades formativas del profesorado en centros específicos de Educación Especial", constituido por una escala tipo Likert cuyas respuestas oscilan entre 1 y 4; siendo 1 "Plenamente de acuerdo", 2 "De acuerdo", 3 "En desacuerdo" y 4 "Totalmente en desacuerdo".

Una vez elaborado el instrumento se procedió a comprobar la fiabilidad y validez del mismo con la intención de conocer si el instrumento utilizado mide lo que se pretende medir y si tiene capacidad para ofrecer información de calidad que permita emitir juicios razonados sobre la práctica educativa (Moreno, 2011). En primer lugar, se procedió a la validez de contenido mediante el sistema de jueces expertos. Para ello se invitó a nueve profesores especialistas en el campo de la Didáctica y la Educación Especial, entre los que había docentes de contrastada 
experiencia en el campo (los seis que participaron también en su aplicación y tres más provenientes de otras universidades). De esta forma, se llevó a cabo una primera versión del cuestionario y se procedió a su depuración en sucesivas rondas en las que se entregaron al equipo investigador las oportunas correcciones. Se tuvo en cuenta en estos análisis la pertinencia de las preguntas, la claridad en el lenguaje usado, la suficiencia del número de ítems y la valoración global de la propuesta. De este modo, la escala quedó formada por sesenta y seis ítems.

A continuación se obtuvieron los resultados derivados del estudio de la dimensionalidad subyacente a las respuestas de los sujetos a la escala administrada. En primer lugar, se determinó el índice de discriminación de los ítems de la escala en donde se aprecian valores muy altos y estables, estando en todos los casos por encima de .98. El estudio de la fiabilidad confirma la alta consistencia interna del instrumento al alcanzar el coeficiente alfa de Cronbach el valor de .984, lo que indica que la escala diseñada es muy fiable. Al presentar la fiabilidad de cada una de las dimensiones del cuestionario, los coeficientes de fiabilidad son bastante altos (oscilan entre .871 y .984), lo que nos permite asegurar que cada una de las dimensiones mide realmente aquello que se pretende medir. Por su parte, para el método de las dos mitades se obtiene para la primera parte un valor de .979 y para la segunda un valor de .976 .

La validez de constructo complementa la validez de contenido llevada a cabo en la fase previa mediante el sistema de jueces expertos, anteriormente expuesta. El objetivo principal es comprobar si los sesenta y seis ítems de la escala se pueden resumir de alguna forma, es decir, si existen rasgos comunes entre ellos. Con tal motivo, se recurre al análisis factorial por el método de los componentes principales como forma de agrupar estos reactivos en factores o componentes que puedan explicar la varianza observada en las respuestas ofrecidas por los sujetos (Rojas, Fernández y Pérez, 1998; Escalante y Caro, 2006). Para ello, se procede a conocer el grado de correlación entre las variables, el cual ha de ser alto para poder realizar el análisis factorial; así pues, el índice de adecuación muestral KMO alcanza un valor de .947 y la prueba de esfericidad de Bartlett $\mathrm{p}<.000$, confirmándose la existencia de correlaciones significativas entre las variables estudiadas. Además, el análisis de los componentes principales revela, tras la rotación Varimax, la convergencia de cuatro factores que explican el 63,452\% de la varianza tales como:

- Factor 1. Desarrollo profesional del docente en centros de Educación Especial.

- Factor 2. Actitudes del docente hacia la inclusión educativa.

- Factor 3. Estrategias didácticas en el proceso de enseñanza-aprendizaje.

- Factor 4. Estructura organizativa de los centros de Educación Especial.

\subsection{Muestra}

Este estudio está dirigido a todos los docentes que durante el curso académico 2010-2011 han desarrollado su labor en alguno de los centros de Educación 
Especial de Andalucía. Según datos aportados por la propia Consejería de Educación de la Junta de Andalucía, han sido 1.060 los docentes encargados de atender a 3.447 alumnos con discapacidad en este tipo de centros. En la comunidad autónoma andaluza existen un total de 59 centros, de los cuales 16 son de titularidad pública (quince gestionados directamente por la Consejería de Educación de la Junta de Andalucía y uno de convenio titularidad del Ayuntamiento de Sevilla). El resto de centros son de titularidad privada, gestionados por entidades suscritas a conciertos con la Administración Educativa.

La muestra a partir de la cual se han establecido las conclusiones del estudio responde a un tipo de muestreo no probabilístico de tipo accidental o casual (Tójar y Matas, 2009) estando formado por todos aquellos docentes que aceptaron participar en la investigación contestando a los cuestionarios enviados $(n=428)$. Dicha muestra preserva el criterio de representatividad, tomando un nivel de confianza del 95\% y un error de estimación máximo del 4\%.

Tal y como se muestra en la Tabla 1, del total de la muestra, un 73,1\% lo componen mujeres, mientras que un $26,9 \%$ son varones. Sus edades oscilan, en un $17,0 \%$ de los casos, entre 41 y 45 años y entre 46 y 50 años, respectivamente; un porcentaje algo menor lo constituyen docentes con edades entre 26 y 30 años $(16,8 \%)$ y mayores de 50 años (16,3\%). En cuanto a su antigüedad y experiencia en centros de Educación Especial, un 29,5\% de la muestra afirma llevar trabajando en este tipo de centros educativos más de 20 años, mientras que un 20,0\% posee una experiencia entre 1 y 3 años, un 16,3\% entre 15 y 20 y un 15,1\% entre 4 y 7 años.

Centrados en la formación académica de la muestra, la gran mayoría son maestros especialistas en Pedagogía Terapéutica (80,3\%), quedando en un segundo plano las titulaciones de maestros, especialistas en Audición y Lenguaje (9,6\%), Pedagogía (4,2\%), Formación Profesional (3,0\%), Psicología (1,2\%), Psicopedagogía $(1,0 \%)$ y Logopedia $(0,7 \%)$. Por su parte, un 68,8\% afirma haber recibido algún tipo de formación práctica sobre educación inclusiva durante su proceso de formación inicial. De igual modo, un 94,7\% mantiene haber realizado actividades de formación permanente vinculadas al ámbito de la educación inclusiva. 
TABLA 1

Descripción de la muestra

\begin{tabular}{|c|c|c|c|}
\hline & & FRECUENCIA & PORCENTAJE \\
\hline \multirow{2}{*}{ Género } & Varón & 115 & 26.9 \\
\hline & Mujer & 313 & 73.1 \\
\hline \multirow{7}{*}{ Edad } & Menos de 25 años & 10 & 2.4 \\
\hline & Entre 26 y 30 años & 71 & 16.8 \\
\hline & Entre 31 y 35 años & 67 & 15.8 \\
\hline & Entre 36 y 40 años & 62 & 14.7 \\
\hline & Entre 41 y 45 años & 72 & 17 \\
\hline & Entre 46 y 50 años & 72 & 17 \\
\hline & Más de 50 años & 69 & 16.3 \\
\hline \multirow{7}{*}{ Titulación } & $\begin{array}{l}\text { Maestro, especialista en } \\
\text { Pedagogía Terapéutica }\end{array}$ & 326 & 80.3 \\
\hline & $\begin{array}{l}\text { Maestro, especialista en } \\
\text { Audición y Lenguaje }\end{array}$ & 39 & 9.6 \\
\hline & Psicología & 5 & 1.2 \\
\hline & Pedagogía & 17 & 4.2 \\
\hline & Psicopedagogía & 4 & 1 \\
\hline & Logopedia & 3 & 0.7 \\
\hline & Formación Profesional & 12 & 3 \\
\hline \multirow{6}{*}{$\begin{array}{l}\text { Antigüedad como docente en } \\
\text { centros de Educación Especial }\end{array}$} & Menos de un año & 26 & 6.3 \\
\hline & Entre 1 y 3 años & 82 & 20 \\
\hline & Entre 4 y 7 años & 62 & 15.1 \\
\hline & Entre 8 y 14 años & 52 & 12.7 \\
\hline & Entre 15 y 20 años & 67 & 16.3 \\
\hline & Más de 20 años & 121 & 29.5 \\
\hline \multirow{2}{*}{$\begin{array}{l}\text { Formación práctica relacionada } \\
\text { con educación inclusiva }\end{array}$} & Sí & 280 & 68.8 \\
\hline & No & 127 & 31.2 \\
\hline \multirow{2}{*}{$\begin{array}{l}\text { Formación permanente sobre } \\
\text { educación inclusiva }\end{array}$} & Sí & 390 & 94.7 \\
\hline & No & 22 & 5.3 \\
\hline
\end{tabular}

\subsection{Análisis de los datos. Tratamiento estadístico}

Tras la cumplimentación del cuestionario por la muestra, se ha llevado a cabo el análisis de datos mediante el paquete informático Statistical Package for the Social Sciences (SPSS versión 20) al considerarlo un recurso idóneo para esta investigación. Así, los resultados aportados en este trabajo revelan los datos obtenidos en uno de los factores del cuestionario centrado en conocer las actitudes del docente de los centros de Educación Especial hacia la inclusión educativa. 
M.․․ DEL CARMEN PEGALAJAR PALOMINO Y M..$^{\underline{a}}$ JESÚS COLMENERO RUIZ ACTITUDES DEL DOCENTE DE CENTROS DE EDUCACIÓN ESPECIAL HACIA LA INCLUSIÓN EDUCATIVA

Así pues, el análisis de datos se ha centrado en un análisis descriptivo de aquellos ítems más significativos del factor así como una prueba de comparación de medias (prueba t de Student para muestras independientes). No obstante, y para determinar si se cumple o no el principio de homocedasticidad, dicho procedimiento ofrece la prueba de Levène sobre igualdad de varianzas, teniendo en consideración que el nivel de significación utilizado es de 0,05.

\section{RESULTADOS}

\subsection{Resultados del análisis descriptivo}

Con la intención de analizar las actitudes del docente de los centros de Educación Especial de Andalucía acerca de la educación inclusiva, se han examinado las puntuaciones de las medias y desviaciones típicas obtenidas en cada uno de los ítems estudiados (Tabla 2). De este modo, los valores de respuesta obtenidos oscilan entre 4 (valor máximo) y 1 (valor mínimo), siendo éstos considerados en el cuestionario como "totalmente en desacuerdo" y "plenamente de acuerdo" respectivamente. Así pues, la inclusión en centros de Educación Especial, lejos de concebirse como medida segregadora de atención a la diversidad, está basada en una cultura de flexibilidad y creatividad continua, apertura permanente al exterior, desarrollo de programas de intervención en la comunidad y actividades y recursos con centros ordinarios.

Los resultados obtenidos demuestran cómo los docentes se han mostrado muy favorables a la hora de considerar cómo la inclusión constituye uno de los pilares básicos de la educación $(\mathrm{M}=1.63$; D. T. $=1.047)$. De igual modo, también muestran su acuerdo para definir los centros de Educación Especial como instituciones tendentes a la mejora de la calidad de vida del alumnado y consecución de un mayor grado de autonomía personal y funcionalidad en las actividades de la vida diaria $(\mathrm{M}=1.71 ; \mathrm{D} . \mathrm{T} .=1.000)$.

Asimismo, sostienen cómo la atención del alumnado que asiste a los centros de Educación Especial debe estar basada en los principios de normalización e inclusión educativa $(\mathrm{M}=1.92 ; \mathrm{D} . \mathrm{T} .=0.971)$ a la vez que subrayan la conveniencia de la especialización de este tipo de centros educativos según las características y/o discapacidades del alumnado atendido $(M=2.02 ;$ D. T. $=1.072)$.

La respuesta educativa ofrecida al alumnado con discapacidades graves y permanentes debe tener en consideración las necesidades del alumnado escolarizado. Así pues, el docente debe saber definir el ámbito en el que el alumno presenta mayores necesidades y/o dificultades y, por tanto, establecer los mecanismos oportunos para dar respuesta a ello. De este modo, y tomando como referencia los ámbitos en los que los alumnos con discapacidades graves y permanentes escolarizados en los centros de Educación Especial presentan mayores necesidades educativas, los encuestados se muestran más favorables al considerar el ámbito de la salud como uno de los prioritarios $(\mathrm{M}=1.94 ; \mathrm{D}$. T. $=0.989)$, seguido del ámbito 
cognitivo $(\mathrm{M}=1.80 ; \mathrm{D} . \mathrm{T} .=0.965)$, ámbito social $(\mathrm{M}=1.72 ; \mathrm{D} . \mathrm{T} .=1.004)$, ámbito conductual $(\mathrm{M}=1.72 ; \mathrm{D} . \mathrm{T} .=0.982)$, ámbito de autonomía e identidad personal $(\mathrm{M}=$ 1.63; D. T. $=1.074) \mathrm{y}$, finalmente, el ámbito comunicativo $(\mathrm{M}=1.62 ; \mathrm{D} . \mathrm{T} .=1.063)$.

TABLA 2

Resultados del análisis descriptivo (media y desviación típica)

\begin{tabular}{|l|c|c|c|c|}
\hline & MíNIMO & MÁXIMO & MEDIA & $\begin{array}{c}\text { DESVIACIÓN } \\
\text { TÍPICA }\end{array}$ \\
\hline $\begin{array}{l}\text { Considero la inclusión como uno de los } \\
\text { pilares básicos en que se ha de fundamentar la } \\
\text { educación }\end{array}$ & 1 & 4 & 1.63 & 1.047 \\
\hline $\begin{array}{l}\text { La atención al alumnado de los centros de } \\
\text { Educación Especial está basada en los principios } \\
\text { de normalización e inclusión educativa }\end{array}$ & 1 & 4 & 1.92 & 0.971 \\
\hline $\begin{array}{l}\text { Elámbito comunicativo es en el que el alumnado } \\
\text { de los centros de Educación Especial presenta } \\
\text { mayores necesidades educativas }\end{array}$ & 1 & 4 & 1.62 & 1.063 \\
\hline $\begin{array}{l}\text { El ámbito de autonomía e identidad personal } \\
\text { es en el que el alumnado de los centros } \\
\text { de Educación Especial presenta mayores } \\
\text { necesidades educativas }\end{array}$ & 1 & 4 & 1.63 & 1.074 \\
\hline $\begin{array}{l}\text { El ámbito social es en el que el alumnado de los } \\
\text { centros de Educación Especial presenta mayores } \\
\text { necesidades educativas }\end{array}$ & 1 & 4 & 1.72 & 1.004 \\
\hline $\begin{array}{l}\text { El ámbito cognitivo es en el que el alumnado } \\
\text { de los centros de Educación Especial presenta } \\
\text { mayores necesidades educativas }\end{array}$ & 1 & 4 & 1.80 & 0.965 \\
\hline $\begin{array}{l}\text { El ámbito de la salud es en el que el alumnado } \\
\text { de los centros de Educación Especial presenta } \\
\text { mayores necesidades educativas }\end{array}$ & 1 & 4 & 1.94 & 0.989 \\
\hline $\begin{array}{l}\text { El ámbito conductual es en el que el alumnado } \\
\text { de los centros de Educación Especial presenta } \\
\text { mayores necesidades educativas }\end{array}$ & 1 & 4 & 1.72 & 0.982 \\
\hline $\begin{array}{l}\text { Creo conveniente la especialización de los centros } \\
\text { de Educación Especial según las características } \\
\text { y/o discapacidades del alumnado atendido }\end{array}$ & 1 & 4 & 2.02 & 1.072 \\
\hline $\begin{array}{l}\text { La escolarización de alumnos en centros de } \\
\text { Educación Especial contribuye a la mejora } \\
\text { de la calidad de vida de éstos aś como a la } \\
\text { consecución de un mayor grado de autonomía } \\
\text { personal y funcionalidad en las actividades de } \\
\text { la vida diaria }\end{array}$ & 1 & 4 & 1.71 & 1.00 \\
\hline
\end{tabular}


M.․․ DEL CARMEN PEGALAJAR PALOMINO Y M. ․ㅡ JESÚS COLMENERO RUIZ ACTITUDES DEL DOCENTE DE CENTROS DE EDUCACIÓN ESPECIAL HACIA LA INCLUSIÓN EDUCATIVA

\subsection{Diferencias según la formación práctica del docente sobre educación inclusiva}

La prueba t de Student para muestras independientes permite comprobar la significación de las diferencias entre grupos, tomando como referencia la variable dicotómica "formación práctica del docente sobre educación inclusiva". Así pues, se presta especial importancia al desarrollo de actividades formativas sobre educación inclusiva por parte del docente del centro de Educación Especial; se trata de una formación de carácter práctico tendente a mejorar y/o actualizar la formación del docente sobre inclusión en el marco escolar.

Por su parte, y con la intención de determinar si se cumple o no el principio de homocedasticidad, la probabilidad asociada al estadístico de Lévene para varianzas iguales para la totalidad de los ítems analizados es, en su mayoría, menor de .05 , con lo que se asume que las varianzas poblacionales no son iguales. Curiosamente, y tras analizar los datos, se confirma cómo las percepciones más favorables entre los docentes se decantan a favor de aquellos que no han realizado ningún tipo de formación práctica vinculada al ámbito de la educación inclusiva durante su período de formación inicial (Tabla 3).

Así pues, los docentes que afirman no haber desarrollado ningún tipo de actividad práctica de carácter formativo sobre educación inclusiva se muestran más favorables al considerar la inclusión como pilar básico de la educación $\left(\mathrm{t}_{402}\right)$ $=-2.53, \mathrm{p}=.000)$ a la vez que sostienen cómo los centros de Educación Especial contribuyen a la mejora de la calidad de vida de éstos así como a la consecución de un mayor grado de autonomía personal y funcionalidad en las actividades de la vida diaria $\left.\left(\mathrm{t}_{399}\right)=-2.35, \mathrm{p}=.000\right)$.

Tomando como referencia las necesidades educativas del alumnado escolarizado en este tipo de centros, los docentes que no han desarrollado ningún tipo de formación práctica vinculada al ámbito de la inclusión se muestran más propicios a considerar el ámbito comunicativo $\left(\mathrm{t}_{391}\right)=-2.09, \mathrm{p}=.000$ ), ámbito de autonomía e identidad personal $\left.\left(\mathrm{t}_{387}\right)=-1.73, \mathrm{p}=.000\right)$, ámbito social $\left(\mathrm{t}_{386}\right)=-1.66$, $\mathrm{p}=.039)$, ámbito cognitivo $\left.\left(\mathrm{t}_{384}\right)=-1.22, \mathrm{p}=.028\right)$ y ámbito conductual $\left(\mathrm{t}_{382}\right)=-.62$, $\mathrm{p}=.011$ ) cómo las áreas en las que los alumnos con discapacidades graves y permanentes precisan de mayores necesidades de intervención docente.

No obstante, no se aprecian diferencias estadísticamente significativas según la formación inicial práctica del docente vinculada al ámbito de la educación inclusiva al valorar cómo la atención del alumnado en este tipo de centros debe estar basada en los principios de normalización e inclusión $\left(\mathrm{t}_{399}\right)=-1.08, \mathrm{p}=.340$ ), la especialización de los centros de Educación Especial según las características del alumnado atendido $\left.\left(\mathrm{t}_{396}\right)=-1.62, \mathrm{p}=.059\right)$ así como la consideración del ámbito de la salud como una de las áreas en las que los alumnos presentan mayores necesidades educativas $\left.\left(\mathrm{t}_{375}\right)=-.73, \mathrm{p}=.071\right)$. 
TABLA 3

Análisis de varianza para la variable "formación práctica sobre educación inclusiva".

Prueba T para muestras independientes

\begin{tabular}{|c|c|c|c|}
\hline \multirow{2}{*}{ FORMACIÓN PRÁCTICA SOBRE EDUCACIÓN INCLUSIVA } & Sí & No & \multirow{2}{*}{ Sig. } \\
\hline & Media & Media & \\
\hline $\begin{array}{l}\text { Considero la inclusión como uno de los pilares básicos en que } \\
\text { se ha de fundamentar la educación }\end{array}$ & 1.68 & 1.40 & $.000^{*}$ \\
\hline $\begin{array}{l}\text { La atención al alumnado de los centros de Educación Especial } \\
\text { está basada en los principios de normalización e inclusión }\end{array}$ & 1.82 & 1.94 & .340 \\
\hline $\begin{array}{l}\text { El ámbito comunicativo es en el que el alumnado de los } \\
\text { centros de Educación Especial presenta mayores necesidades } \\
\text { educativas }\end{array}$ & 1.66 & 1.43 & $.000^{*}$ \\
\hline $\begin{array}{l}\text { El ámbito de autonomía e identidad personal es en el que } \\
\text { el alumnado de los centros de Educación Especial presenta } \\
\text { mayores necesidades educativas }\end{array}$ & 1.66 & 1.46 & $.000^{*}$ \\
\hline $\begin{array}{l}\text { El ámbito social es en el que el alumnado de los centros de } \\
\text { Educación Especial presenta mayores necesidades educativas }\end{array}$ & 1.77 & 1.59 & $.039^{*}$ \\
\hline $\begin{array}{l}\text { El ámbito cognitivo es en el que el alumnado de los centros de } \\
\text { Educación Especial presenta mayores necesidades educativas }\end{array}$ & 1.83 & 1.70 & $.028^{*}$ \\
\hline $\begin{array}{l}\text { El ámbito de la salud es en el que el alumnado de los centros de } \\
\text { Educación Especial presenta mayores necesidades educativas }\end{array}$ & 1.87 & 1.95 & .071 \\
\hline $\begin{array}{l}\text { El ámbito conductual es en el que el alumnado de los centros de } \\
\text { Educación Especial presenta mayores necesidades educativas }\end{array}$ & 1.72 & 1.65 & $.011^{*}$ \\
\hline $\begin{array}{l}\text { Creo conveniente la especialización de los centros de Educación } \\
\text { Especial según las características y/o discapacidades del } \\
\text { alumnado atendido }\end{array}$ & 1.90 & 2.08 & .059 \\
\hline $\begin{array}{l}\text { La escolarización de alumnos en centros de Educación Especial } \\
\text { contribuye a la mejora de la calidad de vida de éstos así como } \\
\text { a la consecución de un mayor grado de autonomía personal y } \\
\text { funcionalidad en las actividades de la vida diaria }\end{array}$ & 1.77 & 1.52 & $.000^{*}$ \\
\hline
\end{tabular}

\section{DisCUSIÓN}

Los resultados obtenidos en la investigación han permitido analizar las percepciones y actitudes de los docentes de los centros de Educación Especial de Andalucía hacia la inclusión educativa. Así pues, lejos de entenderse ésta como una modalidad segregadora, se ha de potenciar su carácter integrador, al plantear actividades y recursos compartidos con centros ordinarios, programas de intervención en la comunidad, apertura permanente al exterior y con una cultura de 
flexibilidad y creatividad continua. Así pues, se puede confirmar cómo, de entre todos los factores determinantes del éxito de la atención a la diversidad, la actitud favorable de los agentes implicados en el proceso aparece como uno de los más importantes (Blas y Calvo, 1995).

Los docentes encargados de ofrecer una respuesta educativa de calidad al alumnado con discapacidad grave y permanente escolarizado en los centros de Educación Especial de Andalucía se muestran favorables al considerar la educación inclusiva como pilar básico de la educación. Estos datos guardan relación con los aportados por Hammod y Lawrence (2003), Kim, Park y Snell (2005) o Batsiou et al. (2008), quienes encontraron actitudes positivas en los docentes hacia la inclusión, entendiendo que ello beneficia a todos los alumnos.

Además, los docentes encuestados definen los centros de Educación Especial como instituciones tendentes a la mejora de la calidad de vida del alumnado y la consecución de un mayor grado de autonomía personal y funcionalidad en las actividades de la vida diaria; en el modelo inclusivo "la educación es un instrumento de promoción y desarrollo personal y social de todos los alumnos" (Pujolás, 2004: 21). Además, y tomando en consideración las aportaciones de Martín y Mauri (2011) aquellos centros que garantizan la inclusión de todos sus alumnos se caracterizan por conseguir calidad en su educación a través de la equidad, acogiendo a toda la población de su entorno, la coordinación y el trabajo colaborativo con toda la comunidad así como la coherencia en sus discursos, prácticas educativas y contenidos a través del consenso y la participación.

No obstante, los encuestados definen los principios de normalización e inclusión como ejes fundamentales para la atención al alumnado con discapacidad grave y permanente, a la vez que consideran necesaria la especialización de este tipo de centros educativos según las características y/o discapacidades del alumnado. El hecho de especializar este tipo de centros educativos según discapacidades permite una atención educativa de más alto nivel, precisando de un conjunto de medios personales y materiales que, por su grado de especialización y elevado coste, únicamente pueden ofertarse de forma conjunta para un limitado número de centros docentes. Para ello, se hace necesario romper con la cultura deficitaria, individual y terapéutica que ha llevado a la marginación y exclusión de estas personas, llegando a un sistema donde los apoyos determinan y delimitan las posibilidades de integración de cualquier persona, promoviendo la idea de que aquello que es positivo para todos se convierte en imprescindible para algunos (Muntaner, 2009).

Tomando como referencia los ámbitos en los que los alumnos con discapacidades graves y permanentes escolarizados en los centros de Educación Especial presentan mayores necesidades educativas, los docentes consideran prioritario el ámbito de la salud, seguido del ámbito cognitivo, social, conductual y, finalmente, el ámbito de la autonomía e identidad personal y comunicativo. Estas áreas de intervención deben tenerse en cuenta para llevar a cabo la práctica educativa en 
M. 므 DEL CARMEN PEGALAJAR PALOMINO Y M. ${ }^{a}$ JESÚS COLMENERO RUIZ

el aula y poder ofrecer al alumnado oportunidades de crecimiento personal según sus necesidades particulares. Para poder construir escuelas inclusivas se necesita alcanzar una concepción inclusiva de la educación, estructurando los centros para incluir a todos los alumnos y que sean capaces de adaptarse a sus características individuales (Ainscow, 2002; Giné, 2008; Larrubia, 2009).

Uno de los retos que plantea el nuevo concepto de educación inclusiva es el relacionado con la formación del profesorado, entendido como un proceso de mejora de los centros educativos y de desarrollo profesional del docente para poder atender las características y necesidades del alumnado (Arnáiz, 2003). Así pues, en esta investigación se ha detectado la existencia de diferencias estadísticamente significativas en las actitudes y percepciones del docente hacia la educación inclusiva en centros de Educación Especial según la formación práctica inicial sobre este ámbito del docente. Así pues, son los docentes que manifiestan no haber desarrollado ningún tipo de actividad práctica durante su período de formación inicial relacionada con la educación inclusiva los que muestran una actitud más favorable hacia la inclusión del alumnado con discapacidad grave y permanente. Estos resultados permiten poner en entredicho la calidad de la enseñanza universitaria y los planes de estudio desarrollados para la formación inicial del docente; así pues, "la enseñanza es vista como un proceso de generar conocimiento con los alumnos, y con los compañeros de la comunidad más inmediata... La mejora de la práctica comienza, entonces, cuando el profesor es capaz de reconceptualizar sus teorías implícitas (creencias y asunciones) de la enseñanza, comprendiendo algunas insuficiencias y limitaciones" (Bolívar, 2000: 53).

Sin embargo, esta circunstancia no suele darse en docentes que inician por primera vez su incorporación a la docencia; se hace necesario, por ello, incardinar los conocimientos que los alumnos traen de la Universidad con la práctica diaria de los centros educativos. Tal y como demuestran Ghanizadeh, Bahredar y Moeini (2006) o Batsiou et al. (2008) existen relaciones significativas entre el conocimiento y la formación docente y sus actitudes hacia la educación inclusiva; así pues, las actitudes negativas del docente hacia la inclusión pueden deberse a carencias formativas en su desarrollo profesional (Gento, 2006).

A modo de síntesis, se puede afirmar que los centros de Educación Especial, junto a las aulas específicas de Educación Especial en centros ordinarios, constituyen un recurso del sistema educativo orientado a lograr que los alumnos con necesidades específicas de apoyo educativo asociadas a discapacidades graves y permanentes alcancen los fines que la educación persigue. La atención al alumnado en este tipo de centros debe desarrollarse desde una perspectiva inclusiva; éstos deben apostar por cumplir una función optimizadora del desarrollo del alumno dirigida a la mejora de la calidad de vida y la consecución de un mayor grado de autonomía y funcionalidad en las actividades de la vida diaria. 


\section{REFERENCIAS BIBLIOGRÁFICAS}

Agran, M.; Alper, S. y Wehmeyer, M. (2002). Access to the general curriculum for students with significant disabilities: What it means to teachers. Education and Training in Mental Retardation and Developmental Disabilities, 37, 123-133.

Aguerrondo, I. (2004). Los desafíos de la política educativa relativos a las reformas de la formación docente. En AA. VV. (Coords.). Maestros en América Latina: Nuevas perspectivas sobre su formación y desempeño (pp. 97-142). Santiago de Chile: PREAL-CINDE.

Ainscow, M. (2002). Rutas para el desarrollo de prácticas inclusivas. Revista de Educación, $327,69-82$.

Ainscow, M.; Booth, T. y Dyson, A. (2006). Improving schools, developing inclusion. Londres: Routledge. http://dx.doi.org/10.1177/1365480206069014

Alghazo, E. y Naggar, E. (2004). General education teachers in the United Arab Emirates and their acceptance of the inclusion of students with disabilities. British Journal of Special Education, 31, 94-99. http://dx.doi.org/10.1111/j.0952-3383.2004.00335.x

Arnáiz, P. (2003). La educación inclusiva: una escuela para todos. Málaga: Aljibe. http://dx.doi.org/10.1080/1360311032000110981.

Avramidis, E. y Kalyva, E. (2007). The influence of teaching experience and professional development on Greek teachers' attitudes towards inclusion. European Journal of Special Needs Education, 22, 367-289. http://dx.doi.org/10.1080/08856250701649989.

Batsiou, S.; Bebetsos, E.; Panteli, P. y Antoniou, P. (2008). Attitudes and intention of Greek and Cypriot primary education teachers towards teaching pupils with special educational needs in mainstream schools. International Journal of Inclusive Education, 12 (2), 201-219. http://dx.doi.org/10.1080/13603110600855739.

Blas, R. y Calvo, I. (1995). Actitudes del profesor ante la integración escolar. En F. Salvador, M. J. León y A. Miñan (Eds.). Integración Escolar: Desarrollo curricular, organizativo y profesional. Actas XII Jornadas Nacionales de Universidades y Educación Especial (pp. 471-480). Granada: ICE Universidad de Granada.

Boer, A.; Jan, S. y Minnaert, A. (2011). Regular primary school teachers'attitudes towards inclusive education: a review of the literature. International Journal of Inclusive Education, 15 (3), 331-353. http://dx.doi.org/10.1080/13603110903030089.

Bolívar, A. (2000). Los centros educativos como organizaciones que aprenden. Promesa y realidades. Madrid: La Muralla.

Booth, T. (2006). Manteniendo el futuro con vida: convirtiendo los valores de la inclusión en acciones. En M. A. Verdugo y F. B. Jordán de Urríes (Coords.). Rompiendo inercias. Claves para avanzar. VI Jornadas Científicas de Investigación sobre Personas con Discapacidad (pp. 211-217). Salamanca: Amarú.

Booth, T. y Ainscow, M. (2000). Guía para la evaluación y mejora de la educación inclusiva. Desarrollando el aprendizaje y la participación en las escuelas. Madrid: Consorcio Universitario para la educación inclusiva.

Bunch, G. (2008). Claves para una educación inclusiva exitosa. Una mirada desde la experiencia práctica. Revista Internacional de Educación Inclusiva, 1, 18-30.

Cardona, M. C. y Gómez, P. F. (2001). Manual de Educación Especial. Valencia: Promolibro.

Colás, M. ․ P. y Buendía, L. (1992). Investigación educativa. Sevilla: Alfar. 


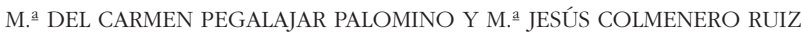

Cole, C. M.; Waldron, N. y Majd, M. (2004). Academic progress of students across inclusive and traditional settings. Mental Retardation, 42, 136-144. http://dx.doi.org/10.1352/0047-6765(2004)42<136:APOSAI>2.0.CO;2

Echeita, G. (2007). Educación para la inclusión o educación sin exclusiones. Madrid: Narcea.

Echeita, G. y Simón, C. (2007). La contribución de la educación escolar a la calidad de la vida de las personas con discapacidad. Ante el reto de su inclusión social. En L. Cayo y R. de Lorenzo (Dirs.). Tratado sobre discapacidad (pp. 1103-1134). Madrid: Aranzadi/ Thomson.

Echeita, G.; Verdugo, M. A.; Sandoval, M.; Simón, C.; López, M.; González-Gil, F. y Calvo, M. I. (2008). La opinión de FEAPs sobre el proceso de inclusión educativa. Siglo Cero, 39 (4), 26-50.

Escalante, E. y Caro, A. (2006). Investigación y análisis estadístico de datos en sPss. Mendoza: Facultad de Educación Elemental y Especial.

FEAPS (2000). Manual de Buena Práctica de Educación. Madrid: FEAPS.

Forlin, C. (2010). Developing and implementing quality inclusive education in Hong Kong: implications for teacher education. Journal of Research in Special Education Needs, 10 (1), 177-184.

http://dx.doi.org/10.1111/j.1471-3802.2010.01162.x

Friend, M. P. y Bursuck, W. D. (2002). Including students with special needs: A practical guide for classroom teachers. Boston: Allyn \& Bacon.

Fullan, M. (2002). Las fuerzas del cambio. Explorando las profundidades de la reforma educativa. Madrid: Akal.

Gento, S. (2006). Propuesta para una acción educativa de calidad en el tratamiento de la diversidad. Revista Complutense de Educación, 17 (2), 13-34.

Ghanizadeh, A.; Bahredar, M. J. y Moeini, S. R. (2006). Knowledge and attitudes towards attention deficit hyperactivity disorder among elementary school teachers. Patient Education and Counseling, 63 (1), 84-88. http://dx.doi.org/10.1016/j.pec.2005.09.002

Giné, C. (2008). El desarrollo local e institucional de proyectos educativos inclusivos. Perspectiva CEP, 14, 17-31.

Hagger, H. y Mcintyre, D. (2006). Learning teaching from teachers. Maidenhead: Open University Press.

Hammond, H. y Lawrence, I. (2003). Teachers' attitudes toward inclusion: survey results from elementary school teachers in three southwestern rural school districts. Rural Special Education Quarterly, 22, 24-30.

Hegarty, S. (2008). Investigación sobre Educación Especial en Europa. Revista Iberoamericana sobre Calidad, Eficacia y Cambio en Educación, 6 (2), 191-199.

Hsien, M. (2007). Teacher attitudes towards Preparation for Inclusion-In support of a unified teacher preparation program. Postgraduate Journal of Education Research, 8 (1), 49-60.

Imbernón, F. (2004). La formación y el desarrollo profesional del profesorado. Barcelona: Graó.

Kalyva, E.; Gojkovic, D. y Tsakiris, V. (2007). Serbian teachers' attitudes towards inclusion. International Journal of Special Education, 22 (3), 31-36.

Kavale, K. (2000). Inclusion: Rhetoric and reality surrounding the integration of students with disabilities. Des Moines: Fine Foundation, the Iowa Academy of Education. 
M. 므 DEL CARMEN PEGALAJAR PALOMINO Y M. ${ }^{a}$ JESÚS COLMENERO RUIZ ACTITUDES DEL DOCENTE DE CENTROS DE EDUCACIÓN ESPECIAL HACIA LA INCLUSIÓN EDUCATIVA

Kavale, K. A. y Forness, S. R. (2000). History, rhetoric, and reality: Analysis of the inclusion debate. Remedial and Special Education, 21, 279-296. http://dx.doi.org/10.1177/074193250002100505.

Kim, J.; Park, E. y Snell, M. E. (2005). Impact of Information and Weekly contact on attitudes of Korean General Educators and Nondisabled students regarding peers with disabilities. Mental Retardation, 43 (6), 401-415. http://dx.doi.org/10.1352/0047-6765(2005)43[401:IOIAWC]2.0.CO;2].

Larrubia, A. (2009). Los preceptos de la escuela inclusiva. Su aplicación en la Educación Secundaria Obligatoria. Temas para la Educación, 3, 1-7.

Lewis, R. B. y Doorlag, H. D. (1999). Teaching special students in general education classrooms. New Jersey: Prentice Hall.

Martín, E. y Mauri, T. (2011). La atención a la diversidad en la escuela inclusiva. En E. Martín y T. Mauri (Coords.). Orientación educativa. Atención a la diversidad y educación inclusiva (pp. 29-49). Barcelona: Graó.

Menter, I.; Hulme, M.; Elliot, D.; Lewin, J. et al. (2010). Literature Review on Teacher Education in the $21^{\circ}$ Century. Scottish Government Social Research.

Moreno, T. (2011). Consideraciones éticas en la evaluación educativa. Revista Iberoamericana sobre Calidad, Eficacia y Cambio en Educación, 9 (2), 130-144.

Muntaner, J. J. (2009). Escuela y discapacidad intelectual. Propuestas para trabajar el aula ordinaria. Sevilla: Eduforma.

Myklebust, J. O. (2007). Diverging paths in upper secondary education: Competence attainment among students with special education needs. International Journal of Inclusive Education, 11, 215-231. http://dx.doi.org/10.1080/13603110500375432.

Orcasitas, J. R. (1994). La investigación en el campo de la Educación Especial y de la educación de deficientes mentales en particular. Revisión de fuentes 1960-1993. San Sebastián: DOE-uPV.

Organización Mundial de la Salud (2011). Informe Mundial sobre Discapacidad. Suiza: OMS.

Orr, A. C. y Hamming, S. (2009). Inclusive postsecondary strategies for teaching students with learning disabilities: A review of the literature. Learning Disability Quarterly, 32, 181-196.

Pujolás, P. (2004). Aprender juntos alumnos diferentes. Los equipos de aprendizaje cooperativo en el aula. Barcelona: Eumo-Octaedro.

Rafferty, Y.; Piscitelli, V. y Boettcher, C. (2003). The impact of inclusion on language development and social competence among preschoolers with disabilities. Exceptional Children, 69, 467-479.

Raffo, C.; Dyson, A.; Gunter, H.; Hall, D.; Jones, L. y Kalambouka, A. (2009). Education and poverty: mapping the terrain and making the links to educational policy. International Journal of Inclusive Education, 13, 341-358. http://dx.doi.org/10.1080/13603110802124462

Rojas, A. J.; Fernández, J. S. y Pérez, C. (1998). Investigar mediante encuestas. Madrid: Síntesis.

Rosenberg, M.; Westling, D. y McLeskey, J. (2008). Special education for today's teachers. Upper Saddle River, NJ: Prentice Hall.

Ruijs, M. N. y Peetsma, T. (2009). Effects of inclusion on students with and without special educational needs reviewed. Educational Research Review, 4, 67-79. http://dx.doi.org/10.1016/j.edurev.2009.02.002. 
Salazar, J. (2010). Modelos teóricos de formación del profesorado. Revisión crítica. En L. Pumares y M. L. Hernández Rincón (Coords.). La formación del profesorado para la atención a la diversidad (pp. 27-50). Madrid: CEP.

Salend, S. J. (1998). Effective mainstreaming. Creating inclusive classrooms. New Jersey: Prentice Hall.

Thomazet, S. (2009). From integration to inclusive education: Does changing the terms improve practice? International Journal of Inclusive Education, 13, 553-563. http://dx.doi.org/10.1080/13603110801923470.

Tójar, J. C. y Matas, A. (2009). Fundamentos metodológicos básicos. En A. Pantoja (Coord.). Manual básico para la realización de tesinas, tesis y trabajos de investigación (pp. 129154). Madrid: EOS.

Vaillant, D. (2005). Formación de docentes en América Latina. Re-inventando el modelo tradicional. Barcelona: Octaedro.

Vezub, L. (2007). La formación y el desarrollo profesional docente frente a los nuevos desafíos de la escolaridad. Profesorado. Revista de Currículum y Formación del Profesorado, 11 (1), 1-23. Descargado el día 15 de octubre de 2013. http://www.ugr.es/ recfpro/rev111ART2.pdf.

Vlachou, A. (2004). Education and inclusive policy-making: implications for research and practice. International Journal of Inclusive Education, 8 (1), 3-21.

http://dx.doi.org/10.1080/1360311032000139449. 